\title{
Anemia in Intensive Care: A review of Current Concepts
}

\author{
Gautam Rawal1², Raj Kumar¹, Sankalp Yadav², Amrita Singh³ \\ 1 Respiratory Intensive Care, Max Super Specialty Hospital, Saket, New Delhi, India \\ 2 Department of Medicine \& TB, Chest Clinic Moti Nagar, North Delhi Municipal Corporation, New Delhi, India \\ ${ }^{3}$ Gen-X Diagnostics, Madhu Vihar, New Delhi, India
}

\begin{abstract}
Anemia in patients admitted to an intensive care unit is common and affects almost all critically ill patients. The intensivist is faced with the challenge of treating multifactorial etiologies, mainly bleeding and blood loss due to phlebotomy and decreased erythropoiesis. Red cell transfusion, the most common treatment for anemia, comes with associated risks, which may further reduce the chance of survival of these patients. The best evidence suggests the practice of restrictive RBC transfusion (transfusion at $\mathrm{Hb}<7 \mathrm{~g} / \mathrm{dl}$ ).

In this article, the etiopathogenesis of the anemia in critically ill is reviewed, and current opinion on the pros and cons of various management strategies are discussed with emphasize on restrictive transfusion policy.
\end{abstract}

Keywords: anemia, critical illness, erythropoietin, intensive care, red blood cell, transfusion

Received: 05 May 2016 / Accepted: 29 June 2016

\section{INTRODUCTION}

Anemia is a common condition associated with critically ill patients admitted to an intensive care unit (ICU). World Health Organization (WHO) defines anemia as a hemoglobin $(\mathrm{Hb})$ level less than $13 \mathrm{~g} / \mathrm{dL}$ (milligram per deciliter) in men and less than $12 \mathrm{~g} / \mathrm{dL}$ in women [1]. Various studies have shown that approximately two-thirds of critical patients admitted to an ICU have a hemoglobin concentration of less than 12 $\mathrm{g} / \mathrm{dl}$ on the day of admission, and $97 \%$ of the patients become anemic after a week in ICU [2,3]. The management of anemia and its impact on critically ill patients has been a matter of debate for some time, and recently, blood product transfusion policies have been changed from liberal transfusion to restrictive transfusion. This review outlines current literature on the etiopathogenesis of anemia in ICUs and their management, as well as transfusion practices and their impact on critically unwell patients.

\section{EtIOPATHOgENESIS OF ANEMIA IN Critically Ill Patients.}

The cause of anemia in critical illness is complex and often multifactorial. Primary mechanisms include:

a. loss of red blood cells (RBCs) due to phlebotomy and bleeding from a surgical site, trauma, venous access site, or gastrointestinal bleed.

b. decreased production of RBCs due to suppression of bone marrow secondary to inflammatory cytokines, drugs, functional or absolute erythropoietin deficiency due to renal dysfunction.

c. nutritional (iron, folic acid, vitamin B 12) deficiency.

d. increased destruction of RBCs (hemolysis) or $\mathrm{RBC}$ precursor in the bone marrow due to toxins and drugs.

(Table 1) [1,3-5].

\section{Blood loss}

Phlebotomy studies have shown that in a critically ill patient admitted to an ICU, the average amount of blood drawn in the course of conducting laboratory

\footnotetext{
Correspondence to: Gautam Rawal, Flat No. 417, Dhruva Apartments, Plot no. 4, I P Extension, Patparganj, Delhi, India, Pin-110092. Tel: +91 1126515050 E-mail: drgautamrawal@hotmail.com

Raj Kumar: Max Super Specialty Hospital, Saket, New Delhi, India, Tel: +91 1126515050.

Sankalp Yadav: Chest Clinic Moti Nagar, North Delhi Municipal Corporation, New Delhi-110015, India. E-mail: drsankalpyadav@gmail.com

Amrita Singh: Gen X Diagnostics, C- 18, Ground Floor, Madhu Vihar, New Delhi- 110092, Tel: 01147529349, 01142175179, 01142175193.
} 
Table 1. Causes of anemia in intensive care

\begin{tabular}{ll} 
Type of Anemia & Causes \\
Blood loss & Phlebotomy \\
& Gastrointestinal bleeding \\
& Trauma \\
& Surgery \\
\hline Erythropoietin & Inflammatory cytokines \\
deficiencies & Renal insufficiency \\
& Drugs \\
& Decrease bone marrow response \\
& (functional deficiency) \\
\hline Nutritional & Low folate levels \\
deficiencies & Low iron levels \\
& Low vitamin B levels (vitamin B12) \\
\hline Hemolysis & Drug reactions \\
& Toxins \\
\hline Coagulation & Sepsis syndrome \\
abnormalities & Thrombocytopenia \\
& Liver disease \\
& Viral infection \\
& Splenomegaly
\end{tabular}

tests, averages about $40-70 \mathrm{ml}$ daily. This amounts to a unit of blood in a week [1,5]. Chant et al. (2006), in a retrospective study, which included 155 patients admitted to a medical or surgical ICU, demonstrated that after twenty-one days, the number of units of RBCs transfused was significantly associated with the daily phlebotomy volume independent of the other confounding factors [6]. This is usually more than the patient can replenish by his or her own production, and predisposes to anemia. This phlebotomy related blood loss has been decreased with the introduction of new conservative phlebotomy techniques which are the use of small volume phlebotomy (pediatric) tubes, minimizing blood loss from the in-dwelling catheters like arterial lines, reinfusion of discarded sample from indwelling lines and reducing blood tests $[2,7]$.

\section{Bleeding}

Active bleeding is responsible for one-third of blood transfusions in critically unwell patients $[3,5]$. Bleeding complications are not limited to the trauma or postsurgical patients. In a prospective cohort study, Arnold et al. (2007) reported that about $90 \%$ of critical patients had bleeding, $38 \%$ at the site of insertion of a vascular catheter, $16 \%$ associated with an endotracheal tube, $15 \%$ at a surgical site and $6 \%$ related to gastrointestinal bleeding [8]. The most prevalent and preventable risk factors for significant bleeding in ICU patients are coagulation abnormalities and stress-induced mucosal lesions [5].

Coagulation abnormalities include thrombocytopenia, consumption of clotting factors, and sometimes disseminated intravascular coagulation (DIC). The causes for thrombocytopenia $[9,10]$, which can occur in approximately $45 \%$ of ICU patients, include:

a. decreased platelet production due to liver disease.

b. increased platelet destruction by an immune response to sepsis.

c. defective bone marrow or viral infection.

d. increased splenic sequestration due to splenomegaly.

e. increased platelet consumption following trauma.

f. hemodilution after a massive RBC transfusion.

g. certain medications.

h. cardiac assist devices.

Although less frequent (1\% incidence), DIC can sometimes occur in patients with severe sepsis $[5,11]$.

Stress-induced upper gastrointestinal bleeding (UGI), either occult or visible, can develop in a patient as a result of physiological stress caused by clinical conditions. It usually manifests in patients who are on a mechanical ventilator for more than forty-eight hours and in patients with coagulopathy $[5,12]$. The incidence of clinically significant gastrointestinal bleeding in mechanically ventilated patients was observed to be $2.8 \%$ $[1,12]$. Other major risk factors of UGI hemorrhage include brain injury, renal failure, liver disease, and gastric ulcers. Effective measures in reducing bleeding associated with the latter condition are by stress ulcer prophylaxis with agents like $\mathrm{H} 2$-receptor antagonists or proton pump inhibitors, and early enteral feeding $[1,12]$.

\section{Defective or decreased erythropoiesis}

Inflammatory cytokines such as tumor necrosis factor- $\alpha$, interleukins, etc. which are released in response to severe sepsis or trauma, cause anemia by several mechanisms. These include inhibition of erythropoietin release from the kidneys, decreased the responsiveness of the bone marrow to erythropoietin, iron sequestration in macrophages, direct suppression of $\mathrm{RBC}$ production and increased destruction of RBCs by macrophages $[4,5,13]$. 


\section{Nutritional deficiency}

A potentially correctible cause of anemia is nutritional deficiency. Rodriguez et al. (2001) showed that in nonbleeding, non-haemolysing medical ICU patients, $13 \%$ patients were nutritionally deficient on day 3 of admission to an ICU. (9\% iron deficiency, $2 \%$ B12 deficiency, $2 \%$ folic acid deficiency) [14].

Woodman et al. (2005) showed that one-third of patients who were 65 years or older had nutritional deficiency anemia. [15]

\section{Iron sequestration}

It has been shown in various studies, that associated with an inflammatory response due to conditions like infections, rheumatologic diseases, inflammatory bowel disease, and critical illness, there is increased level of hepcidin, a liver-produced peptide, which helps in regulating iron metabolism [16,17]. The elevated levels of hepcidin cause increase iron sequestration in macrophages and also reduce iron absorption from the gut,[16-18] leading to iron-restricted erythropoiesis by the body [17,19]. Patteril et al. (2001) and Bellamy et al. (1998) showed that in approximately $35 \%$ of the patients who have anemia at the time of admission to an ICU, the causative factor was iron sequestration $[20,21]$. In some patients, iron deficiency co-exists with inflammation, and the hepcidin levels may be low or variable [17]. Patients having a high hepcidin-level deficiency anemia benefited from intravenous iron therapy with or without erythropoietin stimulating agents.

\section{Drug Reactions}

Drugs can lead to anemia by two primary mechanisms.

a. Suppression of erythropoietin release. Common medicines like calcium channel blockers, theophylline, angiotensin-converting enzyme inhibitors, angiotensin-receptor blockers, and $\beta$-adrenergic blockers may suppress the release of erythropoietin in few patients [5].

b. Hemolysis. Piperacillin, Ceftriaxone, Diclofenac, Methyldopa, Quinine, Primaquine, Sulfa drugs, Nitrofurantoin.

Treatment is by the discontinuation of medication and, if required, using corticosteroids $[5,22]$.

\section{CONSEQUENCES OF ANEMIA}

Anemia is associated with poor outcomes, mostly in elderly patients with chronic disease $[4,5,23]$. The compensatory response of the body in acute or chronic anemia is an extra burden on critical patients, who are often in either newly developed or preexisting cardiopulmonary failure. The body reacts and compensates for anemia by increasing cardiac output (increased heart rate and stroke volume), oxygen unloading (right shift of the oxyhemoglobin dissociation curve) and oxygen extraction. Various studies have shown that anemia is associated with adverse outcomes in acute myocardial infarction, chronic kidney disease, and chronic heart failure $[4,5,23]$. Studies have also shown increased risk of re-intubation or weaning failure from mechanical ventilator in those patients having low hemoglobin levels, more so in patients with chronic obstructive pulmonary disease $[4,5,23]$.

\section{MANAGEMENT OF ANEMIA IN CRITICALLY ILL}

\section{Blood Transfusions}

Transfusion of packed red blood cells (PRBC) in critically ill patients serves to increase oxygen delivery thus decreasing tissue hypoxia. However, many studies have not shown any improvement in oxygen uptake post-transfusion of PRBC. This can be explained by the various adverse factors of stored blood like low levels of 2,3-diphosphoglycerate which decreases the ability of the hemoglobin to unload oxygen to the tissues (shift of oxygen disassociation curve to the left), the structural changes in stored red blood cells resulting in increased aggregation and/or hemolysis, and the accumulation of pro-inflammatory cytokines $[4,5,23,24]$.

\section{口TRANSFUSION-RELATED COMPLICATIONS}

Complications of massive transfusion: A massive transfusion is defined as the replacement of a patient's total blood volume in less than twenty four hours [25]. This can lead to abnormalities of coagulation, serum biochemistry, acid-base balance and temperature homeostasis [25].

\section{Coagulation abnormality}

This occurs as in cases of massive transfusion of PRBC, which lead to dilutional coagulopathy, as the PRBC is 
deficient in platelets and coagulation factors present in plasma. This can be prevented by replacement of clotting factors with transfusions of fresh frozen plasma (FFP), platelets and cryoprecipitate.

Biochemistry: Biochemical complications of massive transfusion include:

a. hypocalcemia due to citrate present as an anticoagulant (acts by binding to the calcium)

b. hyperkalemia.

c. hypomagnesemia.

\section{Acid-base abnormalities}

Stored blood contains citric acid as anticoagulant and lactic acid produced from stored red cells. Citric acid is metabolized by the liver into bicarbonate, which leads to metabolic alkalosis.

\section{Hypothermia}

Can be caused by rapid transfusion of PRBC, which is stored at $4^{\circ} \mathrm{C}[23-25]$.

\section{Transfusion-related acute lung injury (TRALI)}

TRALI is characterized by non-cardiogenic pulmonary edema leading to hypoxia, respiratory distress, and chest X-ray showing new bilateral pulmonary infiltrates. It occurs within minutes to six hours after transfusion and is the most common cause of mortality and morbidity post blood product transfusion. Symptoms may also include fever, tachycardia, hypotension and normal cardiac pressure on invasive monitoring which helps to differentiate from other causes $[24,26,27]$.

Two mechanisms have been proposed to account for the pathogenesis of TRALI:

\section{Immune mediated mechanisms.}

2. Non-immune mediated mechanisms $[26,27]$.

Immune mediated TRALI occurs due to the presence of leukocyte antibodies in the plasma of the donor blood, which are directed against human leucocyte antigens (HLA) and human neutrophil alloantigen (HNA) in the recipient. Non-immune mediation is thought to be as a result of biologically active substances such as lipids and cytokines. Both mechanisms lead to an increased pulmonary microvascular permeability, which is characteristic of TRALI.

Though any blood product can trigger TRALI, it has been commonly associated with transfusion of products containing a high plasma content and the risk increases with the number of transfused products.

\section{Transfusion-associated circulatory overload (TACO)}

Patients at high risk of volume overload (congestive heart failure, low cardiac ejection fraction, renal failure, respiratory failure) may develop TACO on receiving massive or multiple transfusions. Signs and symptoms of TACO are similar to TRALI and include dyspnea, orthopnea, tachypnea, elevated jugular pressures with distended neck veins and increased blood pressure $[24,28,29]$.

\section{Transfusion-related immunomodulation (TRIM)}

This potentially leads to increased risks of nosocomial infection due to the increased levels of mediators in the stored blood, which cause immune suppression in the recipient $[23,24,30]$.

The other potential adverse effects of blood transfusion include transfusion reactions, transfusion-transmitted infections and iron overload due to repeated transfusions of RBCs for the treatment of chronic conditions, resulting in end-organ damage $[23,24]$.

\section{WHEN TO TRANSFUSE BLOOD?}

Various studies and trials have shown that restrictive blood transfusions protocols that use a hemoglobin trigger of $<7 \mathrm{~g} / \mathrm{dL}$ in critically ill patients (except the patients with cardiovascular disease including acute myocardial infarction or unstable angina) have been found to be beneficial in lowering total mortality, infections, and cardiac events [24,31]. A hemoglobin level of $9-10 \mathrm{~g} / \mathrm{dL}$ is aimed for in patients with acute cardiovascular disease and early stages of severe sepsis, $\mathrm{dL}$ $[23,24,31]$.

\section{- Alternatives to Transfusion}

Knowledge of the adverse effects of blood transfusion has focused current research on finding methods to reduce or avoid red blood cell transfusion.

\section{Erythropoiesis-stimulating agents (ESA)}

A review of the literature has shown that in critically ill patients there is an inappropriately low erythropoietin release or a decreased response of the bone marrow to erythropoietin [13,32].

Corwin et al. (2007), in a prospective, randomized, placebo-controlled trial including 146 medical, surgical and trauma patients, concluded that treatment with 
erythropoietin did not help in reducing the rate of red cell transfusions but resulted in a decrease in mortality in trauma patients. Also, the use of erythropoietin was associated with a greater incidence of thrombotic events [33]. This may be due to the facts that ESAs have a delayed effect, or the critical patients have resistance to ESA and require a high dosage, as concluded by Jelkmann et al. (2013) [34]. Thus ESAs are shown to have a beneficial role only in critically ill patients with a concomitant medical disease (renal disease) or in trauma patients $[33,34]$.

\section{Blood Substitutes}

These are also known as oxygen carriers and have not shown promising results being still a subject of research.

The developed oxygen carriers are of two types:

a. Cell-free hemoglobin-based oxygen carriers (HBOCs).

b. Per fluorocarbons.

HBOCs have various adverse effects including nephrotoxicity, impaired perfusion, and increased rates of myocardial infarction and death. Also, their short intravascular half-life of 12 to 48 hours, have limited their use to severe cases of acute bleeding in surgical or trauma patients [4,35-37].

\section{Iron Therapy}

In critically ill patients, there is iron-restricted erythropoiesis due to iron sequestration and decreased absorption from the gut. This suggests a potential, mainly intravenous role, of iron therapy. However, this type of treatment has been challenged as iron has been associated with the growth and virulence of microbes responsible for nosocomial infections and decreasing the iron levels may be a defense response of the body to sepsis $[38,39]$. Further research is needed before this therapy can be accepted on a regular basis.

\section{CONCLUSION}

The occurrence of anemia is almost inevitable in critically unwell patients admitted to an intensive care unit. Considering its multifactorial etiopathogenesis, it has an impact on patient morbidity and mortality. The primary etiologies includes blood loss due to phlebotomy and bleeding and defective erythropoiesis due to the systemic inflammation. Available therapies for improving the survival rates in critically ill patients aim at maintaining adequate oxygenation of tissues, though these therapies are not without their limitations. Blood transfusions are associated with various complications, including an increased risk of ARDS and infections increasing both mortality and morbidity. The potential benefits and the risks involved must be carefully evaluated in every patient for whom a blood transfusion is being considered. The evidence supports the restrictive strategy of RBC transfusion (transfusion at $\mathrm{Hb}<7 \mathrm{~g} / \mathrm{dl}$ ) preferably over a liberal transfusion strategy (transfusion at $\mathrm{Hb}<10 \mathrm{~g} / \mathrm{dl})$, in the majority of the critically ill patients.

The ICU team play a significant role in minimizing blood tests, providing ulcer prophylaxis, early nutrition and adopting restrictive transfusion strategies.

\section{DCONFLICT OF INTEREST}

None declared.

\section{REFERENCES}

1. Astin R, Puthucheary Z. Anaemia secondary to critical illness: an unexplained phenomenon. Extrem Physiol Med. 2014;3:4.

2. Thomas J, Jensen L, Nahirniak S, Gibney RT. Anemia and blood transfusion practices in the critically ill: a prospective cohort review. Heart Lung. 2010;39:217-25.

3. Hajjar LA, Auler Junior JO, Santos L, Galas F. Blood tranfusion in critically ill patients: state of the art. Clinics (Sao Paulo). 2007;62:507-24

4. Athar MK, Puri N, Gerber DR. Anemia and Blood Transfusions in Critically III Patients. Journal of blood transfusion. 2012;2012:629204.

5. McEvoy MT, Shander A. Anemia, bleeding, and blood transfusion in the intensive care unit: Causes, risks, costs, and new strategies. Am J Crit Care. 2013;22:eS1-13.

6. Chant C, Wilson G, Friedrich JO. Anemia, transfusion, and phlebotomy practices in critically ill patients with prolonged ICU length of stay: a cohort study. Crit Care. 2006;10:R140.

7. Harber CR, Sosnowski KJ, Hegde RM. Highly conservative phlebotomy in adult intensive care-a prospective randomized controlled trial. Anaesth Intensive Care. 2006;34:434-7.

8. Arnold DM, Donahoe L, Clarke FJ, et al. Bleeding during critical illness: a prospective cohort study using a new measurement tool. Clin Invest Med. 2007;30:E93-E102.

9. Greinacher A, Selleng K. Thrombocytopenia in the intensive care unit patient. Hematology Am Soc Hematol Educ Program. 2010;2010:135-43.

10. Marks PW. Coagulation disorders in the ICU. Clin Chest Med. 2009;30:123-9. 
11. Levi M, Toh $\mathrm{CH}$, Thachil J, Watson HG. Guidelines for the diagnosis and management of disseminated intravascular coagulation. British Committee for Standards in Haematology. Br J Haematol. 2009;145:24-33.

12. Cook D, Heyland D, Griffith L, Cook R, Marshall J, Pagliarello J. Risk factors for clinically important upper gastrointestinal bleeding in patients requiring mechanical ventilation Canadian Critical Care Trials Group. Crit Care Med. 1999;27:2812-7.

13. Jelkmann I, Jelkmann W. Impact of Erythropoietin on Intensive Care Unit Patients. Transfus Med Hemother. 2013;40:310-8.

14. Rodriguez RM, Corwin HL, Gettinger A, Corwin MJ, Gubler D, Pearl RG. Nutritional deficiencies and blunted erythropoietin response as causes of the anemia of critical illness. J Crit Care. 2001;16:36-41.

15. Woodman R, Ferrucci L, Guralnik J. Anemia in older adults. Curr Opin Hematol. 2005;12:123-8.

16. Lasocki S, Longrois D, Montravers P, Beaumont C. Hepcidin and anemia of the critically ill patient: bench to bedside. Anesthesiology. 2011;114:688-94.

17. Goodnough LT. Iron deficiency syndromes and iron-restricted erythropoiesis (CME). Transfusion. 2012;52:1584-92.

18. Ganz T, Nemeth E. The hepcidin-ferroportin system as a therapeutic target in anemias and iron overload disorders. Hematology Am Soc Hematol Educ Program. 2011;2011:53842.

19. Fernandez R, Tubau I, Masip J, Munoz L, Roig I, Artigas A. Low reticulocyte hemoglobin content is associated with a higher blood transfusion rate in critically ill patients: a cohort study. Anesthesiology. 2010;112:1211-5.

20. Patteril MV, Davey-Quinn AP, Gedney JA, Murdoch SD, Bellamy MC. Functional iron deficiency, infection and systemic inflammatory response syndrome in critical illness. Anaesth Intensive Care. 2001;29:473-8.

21. Bellamy MC, Gedney JA. Unrecognised iron deficiency in critical illness. Lancet. 1998;352(9144):1903.

22. Garratty G. Immune hemolytic anemia caused by drugs. Expert Opin Drug Saf. 2012;11: 635-42.

23. Hayden SJ, Albert TJ, Watkins TR, Swenson ER. Anemia in critical illness: insights into etiology, consequences, and management. Am J Respir Crit Care Med. 2012;185:1049-57.

24. De Souza DA, Silva FG e, Costa PJM de S. Critical evaluation of justifications for the transfusion of red blood cells: the reality of a government emergency hospital. Rev Bras Hematol Hemoter. 2013;35:263-7.
25. Sihler KC, Napolitano LM. Complications of massive transfusion. Chest. 2010;137:209-20.

26. Toy P, Gajic O, Bacchetti P, et al. Transfusion-related acute lung injury: incidence and risk factors. Blood. 2012;119:1757-67.

27. Vlaar AP, Hofstra JJ, Determann RM, et al. The incidence, risk factors, and outcome of transfusion-related acute lung injury in a cohort of cardiac surgery patients: a prospective nested casecontrol study. Blood. 2011;117:4218-25.

28. Narick C, Triulzi DJ, Yazer MH. Transfusion-associated circulatory overload after plasma transfusion. Transfusion. 2012;52:160-5.

29. Li G, Rachmale S, Kojicic M, et al. Incidence and transfusion risk factors for transfusion-associated circulatory overload among medical intensive care unit patients. Transfusion. 2011;51:33843.

30. Vamvakas EC, Blajchman MA. Transfusion-related immunomodulation (TRIM): an update. Blood Rev. 2007;21:32748.

31. Salpeter SR, Buckley JS, Chatterjee S. Impact of more restrictive blood transfusion strategies on clinical outcomes: a metaanalysis and systematic review. Am J Med. 2014;127:124-31.

32. Stubbs JR. Alternatives to blood product transfusion in the critically ill: erythropoietin. Crit Care Med. 2006;34:S160-9.

33. Corwin HL. The role of erythropoietin therapy in the critically ill. Transfus Med Rev. 2006;20:27-33.

34. Jelkmann I, Jelkmann W. Impact of Erythropoietin on Intensive Care Unit Patients. Transfus Med Hemother. 2013;40:310-8.

35. Moore EE, Moore FA, Fabian TC, et al. Human polymerized hemoglobin for the treatment of hemorrhagic shock when blood is unavailable: the USA multicenter trial. J Am Coll Surg. 2009;208:1-13.

36. Natanson C, Kern SJ, Lurie P, Banks SM, Wolfe SM. Cell-free hemoglobin-based blood substitutes and risk of myocardial infarction and death: a meta-analysis. JAMA. 2008;299:230412.

37. Jahr JS, Mackenzie C, Pearce LB, Pitman A, Greenburg AG. HBOC201 as an alternative to blood transfusion: efficacy and safety evaluation in a multicenter phase III trial in elective orthopedic surgery. J Trauma. 2008;64:1484-97.

38. Khan FA, Fisher MA, Khakoo RA. Association of hemochromatosis with infectious diseases: expanding spectrum. Int J Infect Dis. 2007;11:482-7.

39. Weinberg ED. Iron loading and disease surveillance. Emerg Infect Dis. 1999;5:346-52. 